\title{
CERTAIN NUMERICAL RADIUS CONTRACTION OPERATORS
}

\author{
TAKAYUKI FURUTA AND RITSUO NAKAMOTO
}

ABSTRACT. In this paper an operator $T$ means a bounded linear operator on a complex Hilbert space $H$. The numerical radius norm $w(T)$ of an operator $T$, is defined by $w(T)=\sup |(T x, x)|$ for every unit vector $x$ in $H$. An operator $T$ is said to be a numerical radius contraction if $w(T) \leqq 1$. We shall give some theorems on certain numerical radius contraction operators and related results in consequence of these theorems.

Our central result is that an idempotent numerical radius contraction is a projection. Finally we prove that a periodic numerical radius contraction is the direct sum of zero and a unitary operator, that is to say, normal and partial isometric.

We shall begin with the following lemma and its consequence.

LEMMA. If $T$ is idempotent and $w(T) \leqq 1$, then $T$ is a projection.

We shall give two proofs of the Lemma as follows.

First PROOF OF THE Lemma. Let $y=T x$ where $x \in R(T)^{\perp}$. Then for any positive number $t$ we have $T(x+t y)=(1+t) y$ so that

$$
\begin{aligned}
t(1+t)\|y\|^{2} & =((1+t) y, x+t y)=(T(x+t y), x+t y) \\
& \leqq\|x+t y\|^{2}=\|x\|^{2}+t^{2}\|y\|^{2} .
\end{aligned}
$$

Hence $t\|y\|^{2} \leqq\|x\|^{2}$ so that $y=0$. Therefore $T=0$ on $R(T)^{\perp}$ and $T=1$ on $R(T)$ so that $T$ is a projection.

To give the second proof of the Lemma we need the following unpublished paper [8] which is cited with Professor Kato's permission.

THEOREM A. The following two conditions are equivalent

(i) $w(T) \leqq 1$,

(ii) $\left\|e^{z T}\right\| \leqq e^{|z|}$ for all complex numbers $z$.

Proof. Here we cite the proof of [8] for sake of convenience. The condition (ii) is equivalent to $\left\|e^{t\left(e^{i \theta} T-I\right)}\right\| \leqq 1$ for any real $\theta$ and $t \geqq 0$, which is in turn equivalent to that $e^{i \theta} T-I$ is dissipative for any real $\theta$, which is identical with $w(T) \leqq 1$.

Received by the editors July 6, 1970 and, in revised form, September 25, 1970.

AMS 1969 subject classifications. Primary 4710, 4615; Secondary 5230.

Key words and phrases. Complex Hilbert space, bounded linear operator, numerical radius, numerical radius contraction, spectraloid, normaloid, paranormal operator, hyponormal operator, idempotent operator, periodic operator, partial isometric operator. 
Second PRoOf of the Lemma. By the idempotency of $T$ and Theorem A, we have

$$
\left\|I+z T+\frac{z^{2}}{2 !} T^{2}+\cdots\right\|=\left\|I+\left(e^{z}-I\right) T\right\| \leqq e^{|z|} .
$$

Since $z$ is an arbitrary complex number we put $z=t$, where $t$ is a real number. We get the following inequality

$$
\left\|e^{-t} I+\left(1-e^{-t}\right) T\right\| \leqq 1 \text {. }
$$

Thus we can conclude $\|T\| \leqq 1$ as $t \rightarrow \infty$.

Now it is sufficient to show that $T=T^{*} T$; this method is shown in $[4]$.

$$
\begin{aligned}
\left\|T x-T^{*} T x\right\|^{2} & =\|T x\|^{2}-\left(T x, T^{*} T x\right)-\left(T^{*} T x, T x\right)+\left\|T^{*} T x\right\|^{2} \\
& =\|T x\|^{2}-\left(T^{2} x, T x\right)-\left(T x, T^{2} x\right)+\left\|T^{*} T x\right\|^{2} \\
& =\|T x\|^{2}-\|T x\|^{2}-\|T x\|^{2}+\left\|T^{*} T x\right\|^{2} \\
& =\left\|T^{*} T x\right\|^{2}-\|T x\|^{2} \leqq 0
\end{aligned}
$$

therefore $T$ is a projection. In fact it is well known that an idempotent contraction is a projection.

We shall give a generalization, namely, we can weaken the idempotency of the operator in the Lemma as follows.

THEOREM. If $T^{k}=T$ for some integer $k \geqq 2$ and if $w(T) \leqq 1$, then $T$ is the direct sum of 0 and a unitary operator.

Proof. $T^{2(k-1)}=T^{k-2} T^{k}=T^{k-2} T=T^{k-1}$. This implies that $T^{k-1}$ is an idempotent operator; also $w\left(T^{k-1}\right) \leqq(w(T))^{k-1} \leqq 1$ by power inequality [1], [5], [9]. Therefore $T^{k-1}=P$ is a projection by the lemma. Hence we can decompose $T=T_{1} \oplus T_{2}$ where $T_{1}=T \mid R(P)$ and $T_{2}=T \mid N(P)$. Then we have

$$
T=T^{k-1} T=\left(T_{1}^{k-1} \oplus T_{2}^{k-1}\right)\left(T_{1} \oplus T_{2}\right)=(I \oplus 0)\left(T_{1} \oplus T_{2}\right)=T_{1} \oplus 0 .
$$

Now $T_{1}^{k-1}=I$; therefore $w\left(T_{1}^{-1}\right)=w\left(T_{1}^{k-2}\right) \leqq\left(w\left(T_{1}\right)\right)^{k-2} \leqq 1$, so that $T_{1}$ and $T_{1}^{-1}$ have numerical radius $\leqq 1$; hence $T_{1}$ is unitary [10], so the proof is complete.

Corollary 1. If $T^{k}=T$ and $w(T) \leqq 1$, then $T^{k-1}$ is a projection.

This proof is contained in the proof of the Theorem.

We remark that Corollary 1 shows that if $T$ is an idempotent operator that satisfies any of the following conditions: 
(i) $T$ is a contraction,

(ii) Thas equal norm and spectral radius (normaloid [5]),

(iii) $T$ has equal numerical and spectral radius (spectraloid [5]), then $T$ is a projection.

Moreover we remark that Theorem shows that if $T$ is a periodic operator that satisfies any of the above conditions (i), (ii) and (iii), then $T$ is normal and partial isometric.

CoRollary 2 [4]. If $T$ is paranormal (i.e., if $\left\|T^{2} x\right\| \geqq\|T x\|^{2}$ for all unit vectors $x$; see [2], [3], [6], [7]) and if $T^{k}=T$ for some integer $k \geqq 2$, then $T$ is normal and partial isometric.

Proof. If $T$ is paranormal, then $T$ has norm equal to its spectral radius [2], [3], [7]. Hence if $T^{k}=T$ for some $k \geqq 2$, then $T$ is normal and partial isometric by the above remark.

It is known that this class of paranormal operators properly includes that of hyponormal operators $\left(T^{*} T \geqq T T^{*}\right)$ and is properly included in the class of normaloids [2].

We should like to express here our thanks to Professors $\mathrm{M}$. Nakamura and $Z$. Takeda for their kind suggestions in the preparation of this paper and we also should like to make our acknowledgment to Professor T. Kato for his kindly giving us an opportunity to read his manuscript [8] before its publication.

We would like to make our acknowledgment to the referee for his kind advice and valuable comments.

ADDED IN PROOF. A generalization of the results in this paper will appear in the following: T. Furuta, Some theorems on unitary $\rho$ dilations of Sz.-Nagy and Foias (to appear in Acta Sci. Math.).

\section{REFERENCES}

1. C. A. Berger, A strange dilation theorem, Notices Amer. Math. Soc. 12 (1965), 590. Abstract \#625-152.

2. T. Furuta, On the class of paranormal operators, Proc. Japan Acad. 43 (1967), 594-598. MR 36 \#4354.

3. T. Furuta, M. Horie and R. Nakamoto, A remark on a class of operators, Proc. Japan Acad. 43 (1967), 607-609. MR 36 \#4356.

4. T. Furuta and R. Nakamoto, Some theorems on certain contraction operators, Proc. Japan Acad., 45 (1969), 565-567. MR 40 \#6294.

5. P. R. Halmos, Hilbert space problem book, Van Nostrand, Princeton, N. J., 1967. MR 34 \#8178.

6. V. Istratescu, On some hyponormal operator, Pacific J. Math. 22 (1967), 413417. MR 35 \#4747.

7. V. Istratescu, T. Saito and T. Yoshino, On a class of operators, Tôhoku Math. J. (2) 18 (1966), 410-413. MR 35 \#756. 
8. T. Kato, Remarks on the numerical radius (unpublished).

9. C. Pearcy, An elementary proof of the power inequality for the numerical radius, Michigan Math. J. 13 (1966), 289-291. MR 34 \#1853.

10. J. G. Stampfli, Normality and the numerical range of an operator, Bull. Amer. Math. Soc. 72 (1966), 1021-1022. MR 35 \#3470.

Ibaraki University, NaKanarusaWa Machi 4-12-1, Hitachi, Ibaraki, JapaN

Tennoji Senior High School, Osaka, Japan 